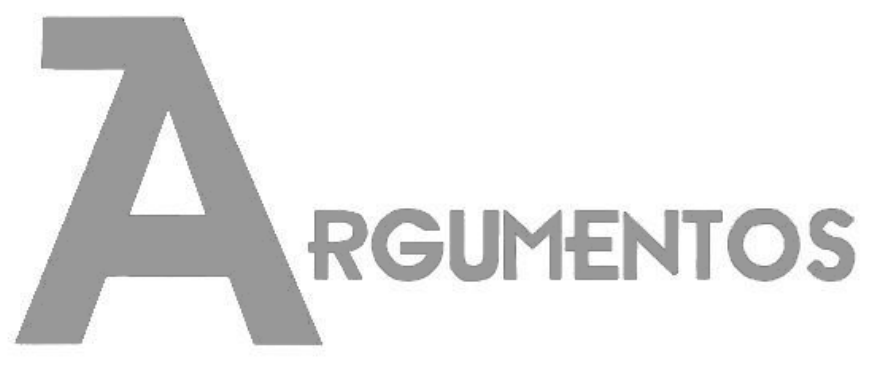

Vol. 18, n. 2, jul./dez. 2021 ISSN: 2527-2551 (online)

\title{
Índice de Desenvolvimento Humano Eficiente e Sustentável (IDHES): Uma Proposta Alternativa
}

\author{
Thiago Costa Soares ${ }^{1}$ \\ Cassiano Ricardo Dalberto ${ }^{2}$ \\ Liana Bohn ${ }^{3}$
}

Recebido em: 23/10/2020

Aprovado em: 21/05/2021

\begin{abstract}
Resumo: O Índice de Desenvolvimento Humano (IDH) é o indicador mais comumente utilizado para se mensurar o nível de desenvolvimento de um país. Contudo, vem sendo alvo de várias considerações na literatura, ensejando propostas alternativas, que incorporem outras dimensões em seu cálculo. $O$ presente trabalho se enquadra nessa linha, objetivando incluir uma medida de eficiência aos componentes do IDH. Tal eficiência diz respeito à utilização de recursos no âmbito da educação e da saúde, e também da emissão de dióxido de carbono (CO2), de modo que países que possuam melhor capacidade de aplicação de fatores produtivos obtenham não somente indicadores de eficiência mais elevados, mas também maiores valores do novo índice de desenvolvimento, o IDHES - Índice de Desenvolvimento Humano Eficiente e Sustentável. Os resultados apontam que poucos países são completamente eficientes, e que os países de maior renda tendem a conseguir alocar melhor os recursos disponíveis, caracterizando um ciclo de reforço positivo ao desenvolvimento no longo prazo - força que ainda precisa ser desencadeada nos países de baixa renda.
\end{abstract}

Palavras-chave: Análise envoltória de dados, meta-fronteira, eficiência ambiental.

Classificação JEL: C14; 01; Q01.

\footnotetext{
1 Professor Adjunto do Departamento de Economia, Universidade Federal de Juiz de Fora, Campus Governador Valadares (UFJF-GV), Brasil. Doutor em Economia, Universidade Federal de Viçosa (UFV/DER), Brasil. E-mail: thiago.costa@ufjf.edu.br. ORCID: https://orcid.org/0000-0002-0117-3785.

2 Professor do Departamento de Ciências Econômicas da Universidade do Estado de Santa Catarina (ESAG-UDESC). Doutor em Economia, Universidade Federal de Minas Gerais (Cedeplar-UFMG), Brasil. Email: cassianord@gmail.com. ORCID: https://orcid.org/0000-0002-6822-6386.

3 Professora Adjunta do Departamento de Economia e Relações Internacionais, Universidade Federal de Santa Catarina (UFSC), Brasil. Doutora em Economia, Universidade Federal de Santa Catarina (UFSC). Email: liana.bohn@ufsc.br. ORCID: https://orcid.org/0000-0003-3443-5048.
} 
Artigo | Índice de Desenvolvimento Humano Eficiente e Sustentável (IDHES): Uma Proposta Alternativa (SOARES, Thiago Costa; DALBERTO, Cassiano Ricardo; BOHN, Liana)

\section{Índice de desarrollo humano eficiente y sostenible (IDH): una propuesta alternativa}

Resumen: El Índice de Desarrollo Humano (IDH) es el indicador más común utilizado para medir el nivel de desarrollo de un país. Sin embargo, para incorporar otras dimensiones en su cálculo, el IDH ha recibido varias consideraciones en la literatura. En este sentido, este trabajo propone incluir un nuevo componente en el IDH para considerar los indicadores de eficiencia técnica en una muestra de 66 países. Para ello, incorporamos al IDH algunas variables que representan la calidad de la asignación de recursos en educación y salud, así como el desempeño en la gestión de las emisiones de dióxido de carbono. Así, los países que tengan mejor capacidad para aplicar recursos en estas áreas podrían obtener un valor más alto en el nuevo índice de desarrollo, el ESHDI - Índice de Desarrollo Humano Eficiente y Sostenible. Los resultados destacaron que unos pocos países son completamente eficientes y los países con mayores ingresos son más eficientes y desarrollados que los demás. Llegamos a la conclusión de que podría existir un ciclo de refuerzo positivo en los países desarrollados, en el que un mayor ingreso podría estimular una mejor asignación de recursos y también el desarrollo humano. Este posible ciclo también debería activarse en los países en desarrollo.

Palabras clave: análisis envolvente de datos, metafrontera, eficiencia ambiental.

Clasificación JEL: C14; 01; Q01.

\section{Efficient and Sustainable Human Development Index (ESHDI): An Alternative Proposal}

Abstract: The Human Development Index (HDI) is the most common indicator used to measure the development level of a country. However, in order to incorporate other dimensions in its calculation, the HDI has been received several considerations in the literature. In this sense, this paper proposes to include a new component into the HDI to consider the technical efficiency indicators in a sample of 66 countries. For this, we incorporated into the HDI some variables that represent the quality of resources allocation in education and health, as well as the performance of managing carbon dioxide emissions. So, the countries that have better ability for applying resources in these areas would be able to obtain a higher value in the new development index, the ESHDI - Efficient and Sustainable Human Development Index. The results highlighted that a few countries are completely efficient, and countries with higher incomes are more efficient and developed than the others. We concluded that might exist a positive reinforcement cycle in the developed countries, in which higher income could stimulate better resource allocation and human development as well. This possible cycle should be also triggered in developing countries. Keywords: Data envelopment analysis, meta-frontier, environmental efficiency.

JEL Classification: C14; 01; Q01.

\section{Introdução}

A construção de indicadores de bem estar é um procedimento habitual em economia para as decisões políticas. Ao longo do tempo, sua aplicação se consolidou e vem se transformando para permitir análises mais realistas do contexto social. Por exemplo, durante muito tempo, a renda, geralmente expressa pelo Produto Interno Bruto (PIB) per capita ou pelo Produto Nacional Bruto (PNB) per capita, desempenhou 
Artigo | Índice de Desenvolvimento Humano Eficiente e Sustentável (IDHES): Uma Proposta Alternativa (SOARES, Thiago Costa; DALBERTO, Cassiano Ricardo; BOHN, Liana)

papel singular como indicador de bem estar (Hicks; Streeten, 1979). Embora ainda seja um dos principais indicadores econômicos da atualidade, sua aplicação como medida única de desenvolvimento recebeu notórias críticas, pois outros importantes aspectos sociais são desconsiderados, como educação e saúde (Stanton, 2007). Nesta perspectiva, surgiram novas ideias para construir indicadores de desenvolvimento mais amplos e representativos.

Sob uma perspectiva histórica, o marco da criação de indicadores de desenvolvimento datou-se em 1966, ano em que foi introduzido o Índice do Nível de Vida, construído pela United Nations Research Institute for Social Development (UNRISD). Logo após, surgiram novas propostas, como o Índice de Desenvolvimento, produzido pela UNRISD, em 1972, e o Índice Físico de Qualidade de Vida (Physical Quality for Life Index-PQLI), criado pelo Overseas Development Council, em 1979. Esses indicadores passaram por um processo de refinamento metodológico, até surgir o Índice de Desenvolvimento Humano (IDH), construído pela ONU, em 1990, o qual se tornou a principal referência na definição de políticas públicas (Dalberto et al., 2015).

Basicamente, o cálculo do IDH envolve três componentes: longevidade, conhecimento e renda. Sua principal contribuição foi a ideia de unir em um único indicador três importantes esferas do desenvolvimento, de forma simples e acessível (Maccari, 2014). Todavia, a composição do índice, desde a sua criação, vem sendo constantemente modificada para lidar não somente com a dinâmica das necessidades sociais, mas também para melhorar a metodologia do procedimento de cálculo 4 .

Em alternativa ao IDH tradicional, diversos indicadores foram propostos para mensurar a qualidade de vida sob a ótica da percepção de felicidade da população, tais como o Global Well-Being Index, Happy Planet Index (HPI), Gross National Happiness (GNH), entre outros (Oliveira et al., 2016). A proposição desses índices está alinhada à ideia de ampliação do conceito de desenvolvimento humano, com o propósito de consolidar uma nova compreensão daquilo que seria mais relevante para a sociedade. A partir disso, pesquisas buscaram introduzir novos conceitos em medidas de desenvolvimento. Para ilustrar, podem-se citar as medidas de desigualdade de renda

\footnotetext{
4 Por exemplo, em 1990, o "nível educacional" foi medido pela "taxa de alfabetização dos adultos". Já em 1994, essa esfera foi representada pela média ponderada da "taxa de alfabetização dos adultos (2/3)" e "média de anos de escolaridade (1/3)".
} 
Artigo | Índice de Desenvolvimento Humano Eficiente e Sustentável (IDHES): Uma Proposta Alternativa (SOARES, Thiago Costa; DALBERTO, Cassiano Ricardo; BOHN, Liana)

(Hicks, 1997), bem estar psicológico (Keynes, 2006), uso do tempo (Ura et al., 2008), boa governança (Dalberto et al., 2015), diversidade ecológica (Türe, 2012), entre outras.

Um dos pontos mais debatidos é a necessidade de agregar os componentes econômicos, sociais e ambientais em um único indicador, de forma a considerar os efeitos adversos do crescimento sobre o meio ambiente (Costantini; Monni, 2005). Este argumento tem como base a definição de "desenvolvimento sustentável", publicada no Relatório Brundtland, em 1987, e discutida na Conferência do Clima no Rio de Janeiro, em 1992, a "Rio-92". Embora não haja total consenso sobre essa definição, de forma intuitiva pode-se entendê-la como a habilidade de uma nação para produzir, respeitando a capacidade de resiliência do meio ambiente. Ao considerar uma variável ambiental no cálculo do IDH, torna-se possível, portanto, incluir no debate a dimensão ambiental do bem estar.

Não obstante, por ser uma fonte de informações crucial para a adoção de políticas públicas, é função do IDH não somente retratar a realidade contextual de um país, mas também considerar o empenho das nações com vistas a práticas mais sustentáveis, pois a dificuldade em gerenciar os recursos pode ser um importante entrave na busca pelo desenvolvimento, sobretudo em economias com maiores restrições orçamentárias. Além disso, a introdução de índices de eficiência na base do cálculo poderia indicar caminhos para melhorar a eficiência na gestão dos recursos, incluindo os naturais.

Neste sentido, na intenção de ampliar a abrangência do IDH, este estudo tem como objetivo incorporar um índice de performance ambiental como medida de gestão da poluição, em uma amostra composta por 66 países. Em adição, reconhecendo a importância de se computar os esforços de cada um para melhorar o desempenho não somente na esfera ambiental, incluem-se indicadores de eficiência dos gastos com educação e saúde. A inclusão destes termos parte do princípio de que países eficientes poderiam reverter investimentos sociais poupados em outras atividades que não são computadas no IDH tradicional, como cultura ou lazer. Ademais, os recursos racionados poderiam inclusive ser úteis para melhorar a qualidade dos serviços já existentes, fato não considerado no cômputo do IDH (Dalberto et al., 2015).

Este artigo está organizado em mais três seções, além desta introdução. A segunda seção apresenta a estratégia metodológica para a criação dos indicadores de 
Artigo | Índice de Desenvolvimento Humano Eficiente e Sustentável (IDHES): Uma Proposta Alternativa (SOARES, Thiago Costa; DALBERTO, Cassiano Ricardo; BOHN, Liana)

eficiência e os procedimentos utilizados para incluí-los no IDH tradicional. Na terceira, apresentam-se resultados, discussões e implicações econômicas do novo índice. A quarta, e última seção, ressalta as principais conclusões do estudo.

\section{Estratégia metodológica}

\subsection{A construção dos indicadores de eficiência}

O presente estudo propõe a criação de um índice de desenvolvimento humano alternativo, baseado no IDH tradicional e na performance para gerir os recursos financeiros destinados à saúde e à educação, bem como as emissões de $\mathrm{CO}_{2}$, em uma amostra composta por 66 países $^{5}$. Inicialmente, calculam-se as performances pelo método "Análise Envoltória de Dados" (DEA, do inglês Data Envelopment Analysis) nos componentes saúde, educação e poluição, separadamente. O DEA permite construir fronteiras de eficiência técnica (fronteiras de possibilidades de produção, FPP), compostas pelas unidades mais eficientes, considerando múltiplos insumos e produtos. A essas observações, são atribuídos índices unitários, enquanto as demais assumem escores inferiores à unidade, que representam sua distância em relação à FPP. Os indicadores de eficiência podem ser entendidos, portanto, como uma medida relativa de desempenho calculada dentro de um espaço amostral.

A construção da fronteira pode ser realizada tanto pela ótica do insumo, onde objetiva-se minimizar a quantidade utilizada de insumos para dado nível produtivo, quanto pela ótica do produto, buscando maximizar a produção, mantendo a dotação de insumos. Nesta abordagem, utiliza-se a orientação a insumo para construir os indicadores de eficiência dos gastos em saúde e educação, admitindo a hipótese de que parte dos recursos financeiros aplicados poderia ser melhor alocada. Na educação, consideram-se, de maneira desagregada, os gastos com educação primária, secundária e terciária. Na saúde, os insumos são os gastos públicos e privados destinados ao setor. Nesses dois casos, os produtos são, respectivamente, o IDH-educação e o IDH-saúde. Em relação ao indicador ambiental, optou-se pela orientação a produto, pois o objetivo

\footnotetext{
${ }^{5} \mathrm{O}$ tamanho da amostra foi definido pela disponibilidade amostral, após o cruzamento dos dados.
} 
Artigo | Índice de Desenvolvimento Humano Eficiente e Sustentável (IDHES): Uma Proposta Alternativa (SOARES, Thiago Costa; DALBERTO, Cassiano Ricardo; BOHN, Liana)

social, neste caso, é maximizar a produção, minimizando a emissão de $\mathrm{CO}_{2}$ (Chiu et al., 2012). Seguindo a literatura internacional, incorporam-se como insumos os fatores capital, trabalho e energia (Chung et al, 1997; Färe et al., 2005; Chiu et al., 2012). Em ambos os modelos, adota-se a superfície de retornos variáveis de escala (RVE) ${ }^{6}$.

A modelagem depende, no entanto, da condição de homogeneidade amostral. Em suma, o DEA, por construção, assume que todas as observações são homogêneas tecnologicamente (Chiu et al., 2012). Todavia, é fácil perceber a existência de diferentes padrões de produção em dados mundiais. No caso ambiental, por exemplo, dados do World Bank (2016) mostram que $68 \%$ da energia elétrica consumida no Brasil provém da produção hidroelétrica (mais limpa), enquanto nos Estados Unidos (EUA), 67\% é de origem fóssil (mais intensiva em emissões). Do mesmo modo, a estrutura das instituições de saúde e de ensino, isto é, máquinas, equipamentos, procedimentos, qualificação profissional etc., pode variar sistematicamente em uma amostra composta por países desenvolvidos e em desenvolvimento, como é o caso da presente pesquisa. Esses fatos sugerem que as unidades possuem dotações tecnológicas diferentes, o que justifica a existência de fronteiras locais. Uma das inovações deste estudo, neste contexto, se dá no tratamento mais adequado desta questão.

Empiricamente, empregou-se a técnica “meta-fronteira”, sugerida por O’Donnell et al. (2008), onde são estimadas fronteiras em grupos previamente divididos por padrões tecnológicos. Esse procedimento garante uma substancial redução dos problemas técnicos relacionados com a heterogeneidade da amostra. Consideram-se $\mathrm{N}$ países, I insumos, D produtos desejáveis e U subprodutos não desejáveis. No caso da saúde e da educação, o conjunto U é vazio, enquanto no ambiental, ele é composto pela emissão de $\mathrm{CO}_{2}$. Sejam $x_{i j}, y_{d j}$ e $b_{u j}$ os valores do i-ésimo insumo $x_{i j}=\left(x_{1 j}, \ldots, \mathrm{x}_{l j}\right) \in \mathfrak{R}_{+}^{I}$ , do d-ésimo produto desejável $y_{d j}=\left(y_{1 j}, \ldots, y_{D j}\right) \in \mathfrak{R}_{+}^{D}$ e do u-ésimo subproduto não desejável $b_{u j}=\left(b_{1 j}, \ldots, b_{U j}\right) \in \mathfrak{R}_{+}^{U}$ para toda $\operatorname{DMU}_{j}\left(j \in \mathfrak{R}_{+}^{N}\right)$. Seguindo Chung et al. (1997), Färe e Grosskopf (2004) e Chiu et al. (2012), assume-se uma função com tecnologia direcional, que permite expandir a cesta de bens desejáveis, minimizando

\footnotetext{
${ }^{6}$ Os detalhes pormenorizados das superfícies em modelos DEA podem ser vistos em Banker, Charnes e Cooper (1984).
} 
Artigo | Índice de Desenvolvimento Humano Eficiente e Sustentável (IDHES): Uma Proposta Alternativa (SOARES, Thiago Costa; DALBERTO, Cassiano Ricardo; BOHN, Liana)

conjuntamente os não desejáveis associados ao processo (quando existem), conforme expressão (1).

$$
\vec{D}^{m}\left(x, y, b, g_{y}, g_{b}\right)=\max \left\{\theta^{m}:\left(x, y+\theta^{m} g_{y}, b-\theta^{m} g_{b}\right) \in T^{m}(x, y, b)\right\}
$$

Nos termos definidos em (1), $g=\left(g_{y}, g_{b}\right)$ representa um vetor direcional diferente de zero, sendo $g \in \mathfrak{R}_{+}^{D} \times \mathfrak{R}_{+}^{U}$. O conjunto denominado "meta-fronteira" é dado por $\mathrm{T}^{\mathrm{m}}=\mathrm{P}(\mathrm{x})^{m}=\{(\mathrm{x}, \mathrm{y}, \mathrm{b}): \mathrm{x}$ produz $(\mathrm{y}, \mathrm{b})\}$. A meta-fronteira compara as práticas produtivas de todos os países e descreve a curvatura eficiente com base nos benchmarks (melhores práticas), considerando apenas uma tecnologia disponível. Após este processo, constroem-se $k$ fronteiras em subgrupos locais divididos por critérios tecnológicos, através de (2).

$$
\begin{aligned}
& \vec{D}^{k}\left(x, y, b, g_{y}, g_{b}\right)=\max \left\{\theta^{k}:\left(x, y+\theta^{k} g_{y}, b-\theta^{k} g_{b}\right) \in T^{k}(x, y, b)\right\} \\
& k=1,2,3 \ldots K
\end{aligned}
$$

O conjunto tecnológico do grupo contém os países que fazem parte do mesmo perfil e é definido por $\mathrm{T}^{k}=\mathrm{P}(\mathrm{x})^{k}=\{(\mathrm{x}, \mathrm{y}, \mathrm{b}): \mathrm{x}$ produz $(\mathrm{y}, \mathrm{b})$ no grupo $\mathrm{k}\}$. O conjunto meta-tecnologia, por seu turno, é composto por $k$ conjuntos $\mathrm{T}^{\mathrm{m}}=\left\{T^{1} \cup T^{2} \cup T^{3} \cup \cdots \cup T^{k}\right\}$. Os indicadores de eficiência de uma $\mathrm{DMU}$ o na metafronteira e na fronteira do grupo, com retornos variáveis de escala, podem ser representados pelas seguintes programações lineares: 


$$
\begin{aligned}
& \max \vec{D}^{m}\left(x_{i o}, y_{i o}, b_{i o}\right)=\theta^{m} \\
& \text { s.t. } \sum_{k=1}^{K} \sum_{n=1}^{N_{k}} \lambda_{n}^{k} x_{i n}^{k} \leq x_{i o}^{k}, \quad \quad \mathrm{i}=1,2, \ldots, \mathrm{I}, \\
& \sum_{k=1}^{K} \sum_{n=1}^{N_{k}} \lambda_{n}^{k} y_{d n}^{k} \geq\left(1+\theta^{m}\right) y_{d o,}^{k} \quad \mathrm{~d}=1,2, \ldots, \mathrm{D}, \\
& \sum_{k=1}^{K} \sum_{n=1}^{N_{k}} \lambda_{n}^{k} b_{u n}^{k}=\left(1-\theta^{m}\right) b_{u o}^{k}, \quad \mathrm{u}=1,2, \ldots, \mathrm{U}, \\
& \sum_{k=1}^{K} \sum_{n=1}^{N_{k}} \lambda_{n}^{k}=1, \quad \mathrm{k}=1,2, \ldots, \mathrm{K}, \\
& \lambda_{n}^{k} \geq 0, \quad \mathrm{n}=1,2, \ldots, \mathrm{N}_{\mathrm{k}} \text {, }
\end{aligned}
$$

em que $\lambda_{n}^{k}$ e $\mu_{n}^{k}$ são as variáveis de decisão dos modelos (3) e (4) que permitem projetar a $\mathrm{DMU}_{\circ}$ para a fronteira de possibilidades de produção, caso $\theta^{m} \neq 1$ e $\theta^{k} \neq 1$, respectivamente. A orientação a insumo para os modelos da saúde e educação pode ser obtida por dualidade. Podem-se definir "eficiência meta-tecnologia" (meta-technology - MEE) e "tecnologia do grupo" (group - technological efficiency: GTE) por MEE $=1-\theta^{m}$ e $G T E=1-\theta^{k}$. Graficamente, a meta-fronteira encobre todas as demais fronteiras dos grupos e representa a tecnologia de produção mais avançada (Figura 1). 


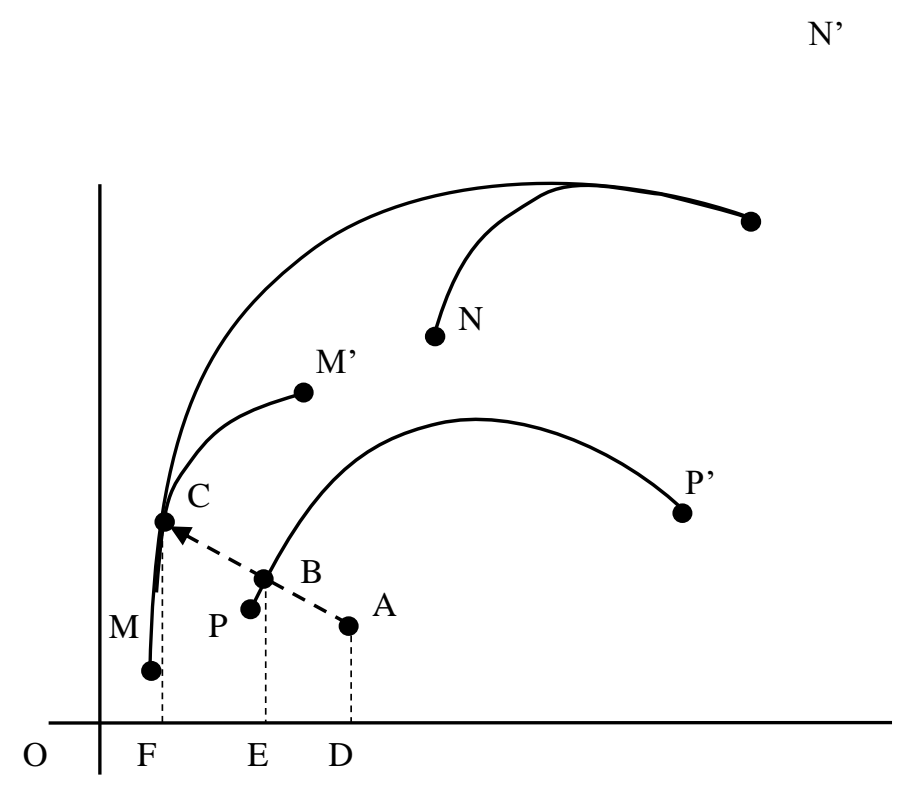

Figura 1. Meta-fronteira e fronteiras dos grupos

Fonte: Adaptado de Chiu et al. (2012)

Neste exemplo, tem-se três grupos distintos. O grupo 1 é composto pelas DMUs $\mathrm{M}$ e $\mathrm{M}^{\prime}$, o grupo 2 por $\mathrm{N}$ e $\mathrm{N}^{\prime}$ e o grupo 3, por A, P e $\mathrm{P}^{\prime}$. As fronteiras eficientes dos grupos são representadas pela ligação dos pontos $M-M^{\prime}, N-N^{\prime}$ e $P-P^{\prime}$, respectivamente. A metafronteira, denotada por $M-N^{\prime}$, envolve todas as fronteiras dos subgrupos. A eficiência da DMU A na meta-fronteira e na fronteira do subgrupo podem ser representadas por MEE $(A)=O F / O D$ e GTE $(A)=O E / O D$, respectivamente. Desse modo, MTR $(A)=O F / O E$. As ineficiências causadas pelo gap tecnológico e pelo gerenciamento inadequado dos recursos são definidas por $\operatorname{TGI}(A)=\operatorname{GTE}(A)(1-M T R(A))=F E / O D$ e $G M I(A)=(1-G T E(A))=$ ED/OD. Assim, define-se ineficiência por MTI $(A)=T G I(A)+G M I(A)=F D / O D$. Observase que a eficiência gerencial está relacionada com a performance quando um país é comparado aos demais do seu grupo. Já a sua eficiência tecnológica diz respeito à distância da meta-fronteira. Os indicadores obtidos em cada grupo farão parte da estrutura do indicador de desenvolvimento proposto.

Para definir os agrupamentos, adotou-se a análise de cluster. Aplicou-se o método hierárquico de Ward, considerando a distância Euclidiana padronizada. A escolha do número de grupos baseou-se nos critérios denominados "pseudo F" e "pseudo T2" (Mingoti; Lima, 2006). Seguindo a literatura da área, as variáveis 
Artigo | Índice de Desenvolvimento Humano Eficiente e Sustentável (IDHES): Uma Proposta Alternativa (SOARES, Thiago Costa; DALBERTO, Cassiano Ricardo; BOHN, Liana)

consideradas proxies tecnológicas foram "exportações per capita de produtos de alta tecnologia (em dólares)", o "percentual dos gastos em pesquisa e desenvolvimento em relação ao Produto Interno Bruto (PIB)" e a "renda per capita (em dólares)" (Chiu et al., 2012; Iyer et al., 2006; Oh; Lee, 2010, Zhang; Choi, 2013). Após os referidos procedimentos, seguiu-se com a construção do indicador de desenvolvimento.

\subsection{O cálculo do Índice de Desenvolvimento Humano Eficiente e Sustentável - IDHES}

A construção do IDH envolve o cálculo da média geométrica das variáveis que fazem parte do indicador. Para manter a estrutura do índice, calculou-se a média geométrica dos indicadores de eficiência da saúde, educação e ambiental. Este procedimento permitiu a inclusão de uma quarta dimensão ao IDH tradicional, a qual representa os esforços envolvidos na busca por melhor desempenho nestas áreas. Assim, a eficiência média de cada país pode ser representada por (5).

$$
E F_{o}=\sqrt[3]{E_{S} \cdot E_{E} \cdot E_{A}}
$$

Em (5), $E_{S}$ representa a eficiência dos gastos em saúde, $E_{E}$ é a eficiência dos gastos educacionais e $E_{A}$ é a eficiência ambiental. Em seguida, incorporou-se a média $E F$ às demais dimensões desagregadas do IDH para se obter um novo indicador, denominado "Índice de Desenvolvimento Humano Eficiente e Sustentável - IDHES", conforme se apresenta na expressão (6).

$$
I D H E S=\sqrt[4]{I D H_{S} \cdot I D H_{E} \cdot I D H_{R} \cdot E F} .
$$

Os três primeiros termos propostos em (6), $I D H_{S}, I D H_{E}$ e $I D H_{R}$, representam índices desagregados de desenvolvimento humano para a saúde, educação e renda, respectivamente. $E F$, como discutido, é a média geométrica da eficiência da saúde, educação e ambiental. A Tabela 1 apresenta uma breve descrição sobre os dados utilizados, bem como suas fontes. 
Artigo | Índice de Desenvolvimento Humano Eficiente e Sustentável (IDHES): Uma Proposta Alternativa (SOARES, Thiago Costa; DALBERTO, Cassiano Ricardo; BOHN, Liana)

Tabela 1 - Descrição das variáveis e fontes

\begin{tabular}{|c|c|c|c|}
\hline Variável & Período & Critérios e unidades & Fonte \\
\hline $\begin{array}{l}\text { Gastos per capita em } \\
\text { educação }\end{array}$ & $\begin{array}{l}2000 / \\
2010\end{array}$ & $\begin{array}{l}\text { Média dos valores anuais, em PPP, para } \\
\text { os gastos públicos e privados em } \\
\text { educação primária, secundária e } \\
\text { terciária }\end{array}$ & UNESCO \\
\hline Gastos per capita em saúde & $\begin{array}{l}2000 / \\
2010\end{array}$ & $\begin{array}{l}\text { Média dos valores anuais, em PPP, para } \\
\text { os gastos públicos e privados em saúde }\end{array}$ & WHO \\
\hline IDH & 2010 & $\begin{array}{l}\text { Valores do índice e de seus } \\
\text { componentes desagregados }\end{array}$ & UNDP \\
\hline Emissões de $\mathrm{CO}_{2}$ & 2010 & Em toneladas & World Bank \\
\hline $\begin{array}{l}\text { Exportações de produtos de } \\
\text { alta tecnologia }\end{array}$ & 2010 & Valores em US\$ per capita & World Bank \\
\hline $\begin{array}{l}\text { Gastos com pesquisa e } \\
\text { desenvolvimento }\end{array}$ & 2010 & Valores em \% do PIB & World Bank \\
\hline PIB per capita & 2010 & Valores em US\$ & World Bank \\
\hline Força de trabalho total & 2010 & Em milhões de trabalhadores & World Bank \\
\hline $\begin{array}{l}\text { Formação bruta de capital } \\
\text { fixo }\end{array}$ & 2010 & Em milhões de US\$ & World Bank \\
\hline Consumo de energia & 2010 & Em Btu & World Bank \\
\hline
\end{tabular}

Notas: United Nations Educational, Scientific and Cultural Organization - UNESCO; World Health Organization - WHO; United Nations Development Programme - UNDP; Central Intelligence Agency - CIA. Fonte: Elaboração própria

\section{Resultados}

Esta seção aborda os principais resultados do estudo. Antes de seguir para as evidências da modelagem e da construção do indicador de desenvolvimento, é importante discutir sobre a formação das fronteiras para uma melhor caracterização. Pelo procedimento de Ward, foram construídos três agrupamentos. Apresentam-se, na Tabela 2, as médias das variáveis utilizadas, por grupo. 
Artigo | Índice de Desenvolvimento Humano Eficiente e Sustentável (IDHES): Uma Proposta Alternativa (SOARES, Thiago Costa; DALBERTO, Cassiano Ricardo; BOHN, Liana)

Tabela 2: Média das variáveis por grupo

\begin{tabular}{ccccc}
\hline $\mathbf{N}$ & Grupo & PIBpc & EXP & P\&D \\
\hline 36 & BB & $9.567,46$ & 67,71 & 43,84 \\
14 & AA & $41.330,76$ & $2.127,91$ & $1.096,93$ \\
16 & AM & $31.943,47$ & 768,44 & 424,36 \\
\hline
\end{tabular}

Nota: PIBpc: Produto Interno Bruto per capita; EXP: Exportação per capita de produtos de alta tecnologia; P\&D: Gasto em pesquisa e desenvolvimento como percentual do PIB.

Fonte: Elaboração própria

Os resultados reportados na Tabela 2 mostram importantes diferenças tecnológicas na amostra. Percebe-se que o primeiro agrupamento (BB - baixa renda e tecnologia) foi formado por países em desenvolvimento, como Brasil, Índia e México, que apresentaram indicadores tecnológicos inferiores aos demais. Os outros dois agrupamentos foram compostos por países desenvolvidos, mas que se diferenciam principalmente pelo nível de exportações de produtos de alta tecnologia e grau de investimentos em pesquisa e desenvolvimento. No grupo denominado "AA - alta renda e alta tecnologia", fazem parte países como Estados Unidos, Japão e Dinamarca. No terceiro agrupamento (AM - alta renda e média tecnologia), Itália, Espanha e Reino Unido são exemplos.

Cada agrupamento representa uma fronteira tecnológica local. A ação subsequente consistiu na estimação de fronteiras de eficiência, considerando ora a amostra global, ora a amostra local. Este procedimento visou diminuir o problema de heterogeneidade amostral, permitindo uma clara separação entre a ineficiência causada pelo gerenciamento dos recursos e pela diferença do padrão tecnológico. As médias e os desvios padrão são apresentados na Tabela 3. O formato da Tabela 3 permite ainda comparar os indicadores de eficiência nos componentes saúde, educação e meio ambiente, separadamente. 
Artigo | Índice de Desenvolvimento Humano Eficiente e Sustentável (IDHES): Uma Proposta Alternativa (SOARES, Thiago Costa; DALBERTO, Cassiano Ricardo; BOHN, Liana)

Tabela 3: Médias e desvios padrão dos indicadores de eficiência, por componente ${ }^{7}$

\begin{tabular}{lcccccc}
\hline \multicolumn{1}{c}{ Componente } & MME & GTE & MTR & TGI & GMI & MTI \\
\hline Saúde & 0,935 & 0,947 & 0,988 & 0,012 & 0,053 & 0,065 \\
& $(0,062)$ & $(0,065)$ & $(0,022)$ & $(0,022)$ & $(0,065)$ & $(0,062)$ \\
Educação & 0,897 & 0,907 & 0,989 & 0,010 & 0,093 & 0,103 \\
& $(0,101)$ & $(0,101)$ & $(0,021)$ & $(0,020)$ & $(0,101)$ & $(0,101)$ \\
Meio ambiente & 0,829 & 0,898 & 0,921 & 0,068 & 0,102 & 0,170 \\
& $(0,172)$ & $(0,139)$ & $(0,101)$ & $(0,093)$ & $(0,139)$ & $(0,172)$ \\
\hline
\end{tabular}

Nota: $\mathrm{MME}$ = Eficiência estimada na meta-fronteira; GTE = Eficiência estimada nas subamostras; MTR = Taxa meta-fronteira, TGI = Ineficiência causada pelo fator tecnológico, GMI = Ineficiência causada pelo gerenciamento dos recursos, MTI = Ineficiência estimada na meta-fronteira. Os desvios-padrão encontram-se entre parênteses.

Fonte: Elaboração própria.

Em valores médios, percebe-se que o padrão tecnológico pode afetar a eficiência, sobretudo no modelo ambiental (note-se que a taxa meta-fronteira foi inferior à unidade, indicando diferenças tecnológicas). Não obstante, os resultados sugerem que a principal causa de ineficiência é o gerenciamento inadequado dos recursos, pois, em todos os segmentos, o indicador GMI foi superior ao TGI. Nos segmentos "saúde" e "educação", essas diferenças são ainda mais acentuadas.

Individualmente, os países analisados mostraram-se mais eficientes no gasto com saúde (em seguida, vêm o gasto em educação e a gestão de poluentes). Na saúde, 17, dos 66 países analisados, apresentaram escores de eficiência iguais a um, global e localmente. Por esta razão, podem ser considerados benchmarks no setor. Contudo, é importante ressaltar que a metodologia empregada não considera um limiar mínimo para o indicador de qualidade da saúde (IDH-saúde). Dessa forma, é possível que países de baixo desenvolvimento neste componente façam parte do rol dos mais eficientes simplesmente pelo fato de apresentarem baixa aplicação de recursos financeiros. Este é o caso particular de Burundi, país situado na área central do continente africano. De fato, a eficiência retratada neste estudo é meramente uma questão técnica. Por isso, os países nesta situação deveriam se preocupar em expandir os investimentos, de forma eficiente, para melhorar a qualidade do indicador de saúde. Veja-se, por exemplo, os casos do Japão, Austrália e Nova Zelândia, que foram eficientes com elevado IDH-saúde.

Podem-se extrair justificativas semelhantes para o caso da educação. Entre as 18 nações eficientes neste segmento, notam-se grupos distintos compostos por países de alto e baixo nível educacional (desenvolvidos e em desenvolvimento). A escassez de

\footnotetext{
${ }^{7}$ Os indicadores de (in)eficiência estão em um intervalo [0,1].
} 
Artigo | Índice de Desenvolvimento Humano Eficiente e Sustentável (IDHES): Uma Proposta Alternativa (SOARES, Thiago Costa; DALBERTO, Cassiano Ricardo; BOHN, Liana)

investimentos no setor é, também, a principal causa do surgimento de países com baixa qualidade entre os eficientes, como Nepal, Madagascar e, novamente, Burundi. Entre os países de melhor desempenho e com alto índice educacional, aparecem Nova Zelândia, Noruega e Irlanda.

Em relação ao indicador de eficiência ambiental, percebeu-se uma grande elevação do número de eficientes quando o fator tecnológico foi considerado. $\mathrm{Na}$ fronteira global, 17 países aparecem com escores unitários, dos quais 10 são desenvolvidos. Em perspectiva local, isto é, comparando os países com seus pares de perfil semelhante, este número aumentou para 28, sendo a metade deles desenvolvidos. Especificamente, os dados revelam que o padrão tecnológico foi a principal causa de ineficiência ambiental em 23 países. Deste montante, a maioria é subdesenvolvida, sugerindo que a limitação tecnológica pode ser um importante entrave para o desenvolvimento sustentável. Todavia, é importante ressaltar que o indicador de eficiência ambiental não pode, novamente, ser entendido como um índice estrito de qualidade, pois existe uma grande variedade de estruturas produtivas na composição da fronteira global. Alguns países desenvolvidos, por exemplo, apesar de possuírem uma matriz energética relativamente "suja", se situam sobre a fronteira, pois conseguem "compensar" as emissões através da renda. Em outra direção, muitos países subdesenvolvidos foram eficientes, mas com baixos níveis de renda e estoque de capital. Estas situações não deveriam ser base para políticas econômicas e ambientais a nível mundial. A melhoria tecnológica, por outro lado, parece ser uma estratégia promissora para um avanço no desempenho ambiental. A performance dos grupos, por segmento, pode ser visualizada pela Tabela 4.

Tabela 4 - Ineficiência gerencial e tecnológica nos segmentos da saúde, educação e ambiental, por grupo

\begin{tabular}{|c|c|c|c|c|c|c|}
\hline \multirow[b]{2}{*}{ Grupos } & \multicolumn{3}{|c|}{ Ineficiência gerencial } & \multicolumn{3}{|c|}{ Ineficiência tecnológica } \\
\hline & Saúde & Educação & Ambiental & Saúde & Educação & Ambiental \\
\hline BB & 0,080 & 0,107 & 0,119 & 0,082 & 0,001 & 0,098 \\
\hline AA & 0,035 & 0,052 & 0,071 & 0,035 & 0,031 & 0,009 \\
\hline AM & 0,009 & 0,096 & 0,091 & 0,051 & 0,014 & 0,052 \\
\hline
\end{tabular}

Nota: $\mathrm{BB}=$ Baixa renda e tecnologia; $\mathrm{AA}$ = Alta renda e tecnologia; $\mathrm{AM}=$ Alta renda e média tecnologia Fonte: Elaboração própria 
Artigo | Índice de Desenvolvimento Humano Eficiente e Sustentável (IDHES): Uma Proposta Alternativa (SOARES, Thiago Costa; DALBERTO, Cassiano Ricardo; BOHN, Liana)

Os resultados expostos na Tabela 4 destacam melhor as diferenças entre os grupos e podem sugerir caminhos que os países poderiam adotar para reduzir seus problemas nestes setores. Em particular, veja-se o caso dos países em desenvolvimento (grupo BB), que apresentaram maiores níveis de ineficiência, tanto gerencial quanto tecnológica. No caso particular da saúde, a combinação entre a expansão dos recursos com a melhoria de sua aplicação poderia contribuir para gerar resultados mais satisfatórios. Já na educação, os resultados sugerem que a qualidade do gasto já existente é a principal ferramenta para melhorar o IDH-educação. Os países desenvolvidos (AA e AM), por sua vez, não apresentaram grandes problemas gerenciais e tecnológicos na saúde. Na educação, porém, as evidências indicam que os gastos poderiam ser melhor aplicados. No caso ambiental, todos os agrupamentos apresentaram elevados níveis de ineficiência, principalmente o grupo dos países em desenvolvimento. Conforme fora discutido acima, a ineficiência deste segmento pode ser explicada, em grande parte, pelas diferenças observadas na matriz energética e pelas várias formas de "compensar" as emissões de poluentes. Os Estados Unidos, por exemplo, apesar de serem o segundo maior poluidor em escala global, foram eficientes, pois possuem a maior renda mundial. No extremo oposto, países como El Salvador, Quênia e Panamá foram eficientes, mas apresentando níveis substancialmente inferiores de capital e renda. Por isso, é importante ressaltar que os argumentos expostos acima são, na realidade, uma generalização. Em termos individuais, os países revelam necessidades particulares e a situação dos três setores deve, por isso, ser analisada caso a caso.

Após calculados os indicadores de eficiência de cada dimensão (saúde, educação e ambiental), obteve-se o indicador de eficiência média, dado pela média geométrica destas três eficiências. A eficiência média permite verificar quais países tiveram melhor desempenho geral na gestão de seus recursos e que serão, posteriormente, menos penalizados pelo desperdício no cômputo do indicador final. A Tabela 5 apresenta a média geral e por grupos tecnológicos do indicador de eficiência média. 
Artigo | Índice de Desenvolvimento Humano Eficiente e Sustentável (IDHES): Uma Proposta Alternativa (SOARES, Thiago Costa; DALBERTO, Cassiano Ricardo; BOHN, Liana)

Tabela 5 - Eficiência média da amostra e por grupos tecnológicos

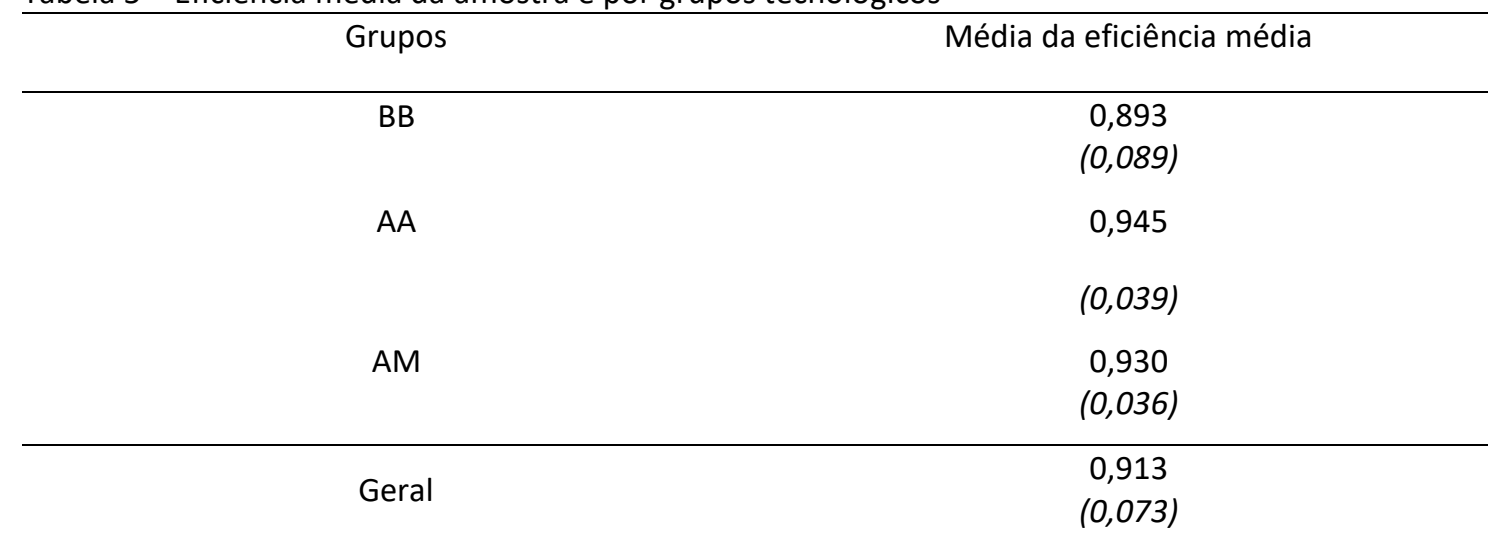

Nota: $\mathrm{BB}$ = Baixa renda e tecnologia; $\mathrm{AA}$ = Alta renda e tecnologia; $\mathrm{AM}$ = Alta renda e média tecnologia. Os desvios-padrão encontram-se entre parênteses.

Fonte: Elaboração própria

Os países de baixa renda e baixa tecnologia apresentaram, de maneira geral, os menores indicadores médios de eficiência. Não obstante, os quatro países que atingiram a maior eficiência média (Burundi, Cuba, Madagascar e Nepal, todos com valor igual a 1) pertencem a tal grupo. Por outro lado, esse resultado é contrastado pelo fato de que os dez países mais ineficientes também pertencem a esse grupo (dentre os quais os quatro piores são Senegal, Índia, Burkina Faso e Lesoto). Essas diferenças se refletem na magnitude do desvio-padrão, como é possível observar acima, e revelam uma grande heterogeneidade entre os países em desenvolvimento no que tange à melhor aplicação de seus recursos.

Os maiores valores médios de eficiência tendem a pertencer ao grupo de alta renda e tecnologia, sendo que o grupo de alta renda e média tecnologia ocupa uma posição intermediária. Somados aos resultados do agrupamento BB, essa constatação sugere a existência de uma relação positiva entre o nível de desenvolvimento dos países e suas respectivas capacidades de aplicação de recursos. Em outros termos, o desenvolvimento de um país pode estar alinhado ao ambiente de maior eficiência tecnológica e de práticas de gestão. Isso, por seu turno, aponta para a possibilidade de ciclos virtuosos ou viciosos no processo de desenvolvimento: maior eficiência abre caminho para obter novos aprimoramentos nas áreas consideradas, que permitirão, posteriormente, melhorar ainda mais a capacidade de utilização eficiente dos fatores. Por outro lado, a ineficiência conduz ao desperdício de insumos que poderiam ajudar a incrementar a qualidade de vida dos países, impedindo-os de obter acesso a tecnologias 
Artigo | Índice de Desenvolvimento Humano Eficiente e Sustentável (IDHES): Uma Proposta Alternativa (SOARES, Thiago Costa; DALBERTO, Cassiano Ricardo; BOHN, Liana)

e níveis educacionais necessários para intensificar a racionalização dos recursos. A relação geral da eficiência média com o nível de desenvolvimento dos países pode ser observada nas Figuras A1 e A2 dos Apêndices, que apresentam regressões simples do IDH e do PIB per capita contra a eficiência média.

Ainda, tais relações não podem ser dissociadas das estruturas institucionais, cujos desenvolvimentos estão sujeitos a condições lock-in, isto é, situações onde as estruturas de incentivos vigentes oferecem alta resistência a mudanças (North, 1990). É o exemplo do carbon lock-in, no caso das energias baseadas em combustíveis fósseis: os retornos crescentes destas no presente criam barreiras à difusão de formas alternativas de baixa emissão de carbono, que seriam mais vantajosas no longo prazo (Unruh, 2000).

Na última etapa, o indicador de eficiência média foi reunido aos demais indicadores desagregados do IDH (saúde, educação e renda) para compor uma nova média geométrica, que consiste no Índice de Desenvolvimento Humano Eficiente e Sustentável (IDHES). Devido ao fato de o componente da eficiência média possuir, em geral, valor maior do que o dos indicadores das dimensões do IDH, ele tende a elevar a média geométrica obtida em relação ao IDH, fazendo com que o IDHES apresente valores maiores do que o índice original. Essas informações podem ser observadas na Tabela 6, onde se apresentam as médias desses índices.

Tabela 6 - Eficiência média da amostra e por grupos tecnológicos

\begin{tabular}{ccc}
\hline Grupos & Média do IDHES & Média do IDH \\
\hline BB & 0,703 & 0,653 \\
& $(0,119)$ & $(0,134)$ \\
AA & 0,911 & 0,900 \\
& $(0,018)$ & $(0,016)$ \\
AM & 0,866 & 0,846 \\
& $(0,047)$ & $(0,056)$ \\
\hline Geral & 0,787 & 0,752 \\
& $(0,130)$ & $(0,151)$
\end{tabular}

Nota: $\mathrm{BB}$ = Baixa renda e tecnologia; $\mathrm{AA}$ = Alta renda e tecnologia; $\mathrm{AM}=$ Alta renda e média tecnologia. Os desvios-padrão encontram-se entre parênteses.

Fonte: Elaboração própria

Os maiores incrementos do IDHES em relação ao IDH ocorrem para o grupo de baixa renda e tecnologia, cujos países ganharam, em média, 0,5 pontos no novo índice. 
Artigo | Índice de Desenvolvimento Humano Eficiente e Sustentável (IDHES): Uma Proposta Alternativa (SOARES, Thiago Costa; DALBERTO, Cassiano Ricardo; BOHN, Liana)

Os ganhos do agrupamento AM são, em média, de 0,2 pontos, enquanto que para os países do grupo AA essa diferença é de 0,1 pontos. Esse resultado, entretanto, não se deve à maior eficiência média dos países situados no agrupamento $\mathrm{BB}$, haja vista as informações apresentadas na Tabela 5. Deve-se, na verdade, ao fato de que nesses países os índices desagregados para saúde, educação e renda tenderem a ser consideravelmente menores do que o componente da eficiência, de modo que a inserção deste tenha maior impacto sobre a nova média obtida, em comparação aos países dos demais agrupamentos, cujos indicadores do IDH têm valores mais próximos da eficiência média. Como resultado secundário disto, observa-se uma redução na diferença dos indicadores de desenvolvimento entre os países, o que pode ser verificado pelo menor desvio-padrão do novo indicador. A distribuição do IDHES comparada com o IDH pode ser visualizada na Figura 2.

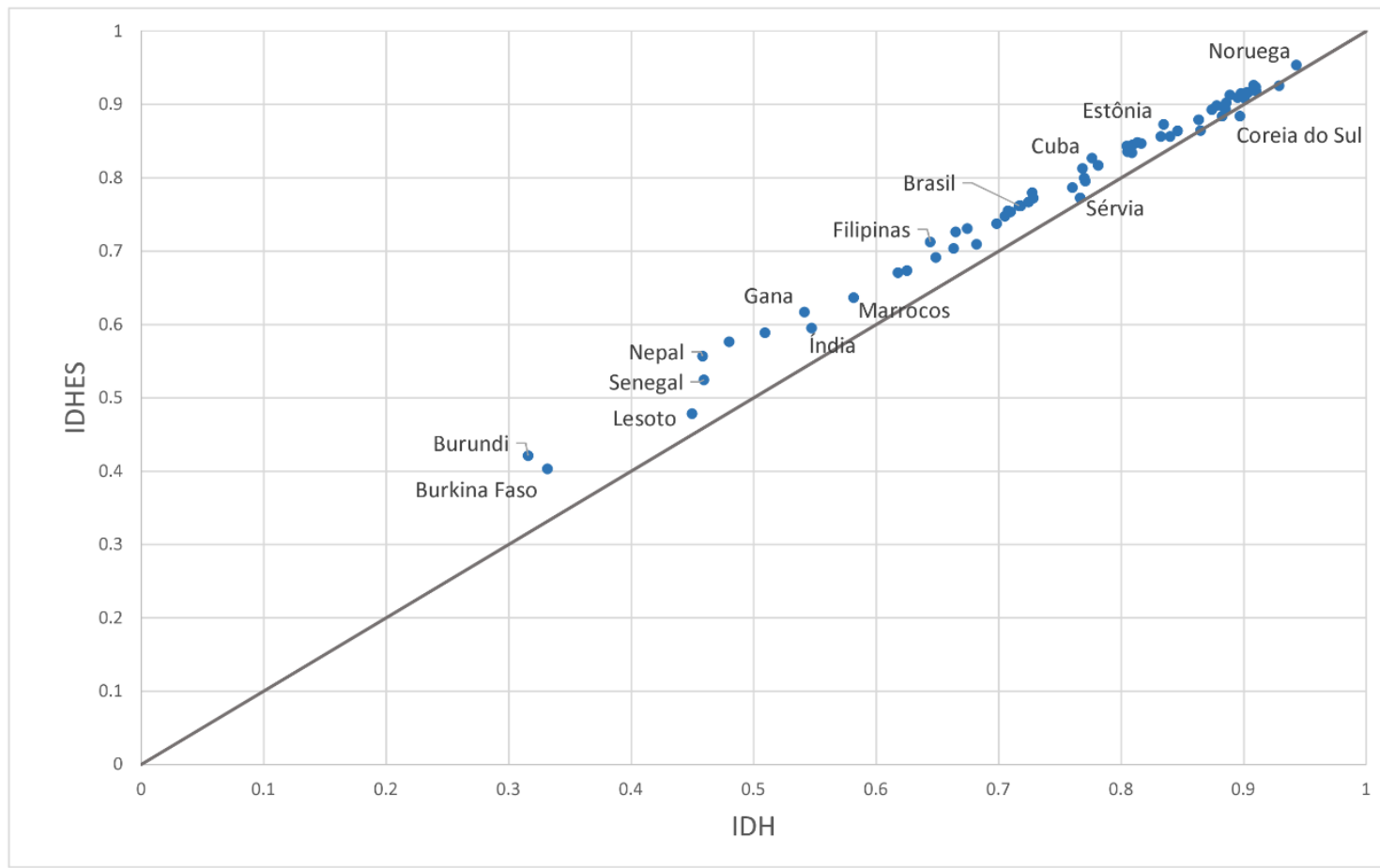

Figura 2. IDHES versus IDH

Fonte: Elaboração própria.

A linha de 45으 na figura representa pontos em que o IDH seria igual ao IDHES, o que ocorreria em casos em que a eficiência média fosse idêntica ao valor do IDH. Como em praticamente todos os casos o componente da eficiência é maior do que tal índice, os pontos que representam os países ficam acima da linha, ilustrando a diferença 
Artigo | Índice de Desenvolvimento Humano Eficiente e Sustentável (IDHES): Uma Proposta Alternativa (SOARES, Thiago Costa; DALBERTO, Cassiano Ricardo; BOHN, Liana)

resultante no IDHES em relação ao indicador original: quanto maior a distância vertical para a linha, maior o ganho proporcionado pela eficiência. Nesse sentido, os países com maiores incrementos foram Burundi, Nepal e Madagascar, todos com eficiência média máxima, cujos ganhos foram de 0,105, 0,099 e 0,097 pontos, respectivamente. Por outro lado, apenas três países apresentaram IDHES abaixo do IDH: República Checa, Austrália e Coréia do Sul, que perderam 0,0003, 0,003 e 0,012 pontos no novo indicador.

Também é possível observar que, embora Nepal e Lesoto tenham valores de IDH bastante próximos $(0,458$ e 0,450, respectivamente), o primeiro país leva considerável vantagem no IDHES $(0,557$, contra 0,478 do Lesoto), justamente por ser um dos países mais eficientes em toda a amostra. Comparação semelhante - que também pode ser visualizada na imagem - pode ser feita entre Cuba e Sérvia: enquanto a diferença nos IDHs de ambos é de apenas 0,01 pontos $(0,776$ contra 0,766 , respectivamente), no IDHES a diferença em favor de Cuba é de 0,054 pontos $(0,827$, contra 0,773 da Sérvia).

No caso do Brasil, o incremento ocorrido no IDHES em relação ao IDH foi de 0,044 (de 0,718 para 0,762), o que se deu por seu indicador de eficiência média ser de 0,910 - valor que o situa em 45 nesse quesito, entre os 66 países da amostra. Em termos de posição nos índices, a situação do país dentro da amostra não se alterou, sendo o 43 은 tanto no IDH quanto no IDHES. Caso o país tivesse obtido eficiência média máxima, seu valor no IDHES seria de 0,780, o que o situaria em 39 na amostra, próximo de Malásia, Bulgária e México. Essa diferença é uma medida do ganho em desenvolvimento que seria possível no curto prazo, como reflexo único da melhor utilização dos recursos nas dimensões consideradas. A Figura 3 apresenta essa relação para todos os países da amostra, contrapondo o IDHES obtido por cada um àquele que seria atingido em caso de eficiência máxima. 
Artigo | Índice de Desenvolvimento Humano Eficiente e Sustentável (IDHES): Uma Proposta Alternativa (SOARES, Thiago Costa; DALBERTO, Cassiano Ricardo; BOHN, Liana)

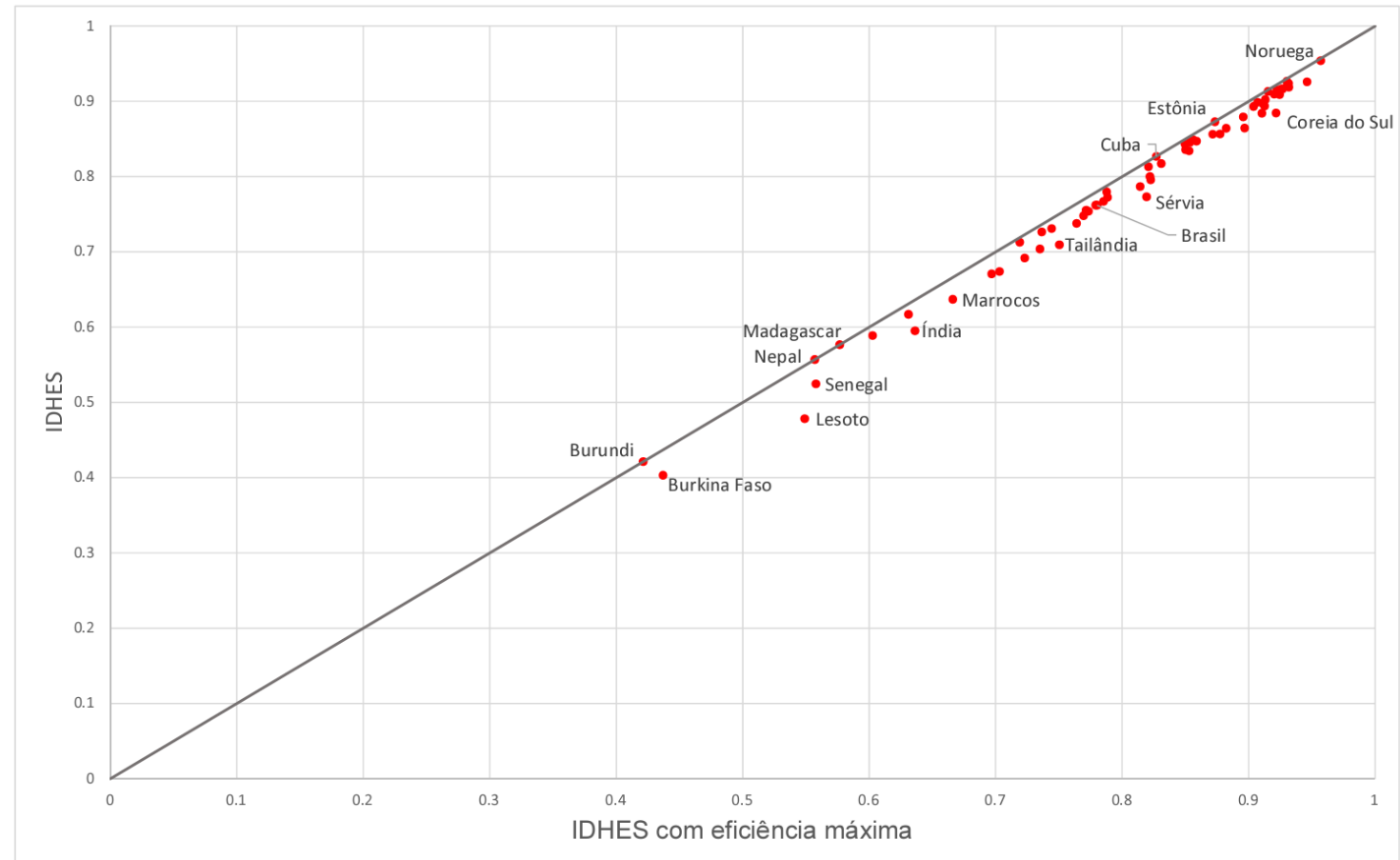

Figura 3. IDHES versus IDHES em caso de eficiência máxima Fonte: Elaboração própria.

Neste caso, a linha de 45 representa os pontos em que os países se situariam atualmente caso tivessem obtido indicador de eficiência igual a 1, de modo que apenas os países de eficiência máxima estão sobre a mesma. Para os demais países, a distância vertical entre seus pontos e a linha indica o impacto da ineficiência no curto prazo: quanto maior esta distância, maior seria o ganho no indicador possível de ser obtido no presente pela melhor aplicação dos recursos nas áreas ambiental, de educação e saúde. É possível notar que algumas das nações que mais teriam a ganhar nesse sentido se situam em estágio de desenvolvimento. A falta de informações para outros países desses grupos, entretanto, não permite verificar se essa tendência é mais ampla. Ainda assim, não é possível desprezar o impacto potencial de uma maior eficiência sobre a capacidade de desenvolvimento dos países, principalmente em uma ótica de longo prazo - aspecto que ainda carece de investigações -, onde a sustentabilidade ambiental e financeira são fatores-chave para a promoção bem-sucedida do aumento de bem estar. 
Artigo | Índice de Desenvolvimento Humano Eficiente e Sustentável (IDHES): Uma Proposta Alternativa (SOARES, Thiago Costa; DALBERTO, Cassiano Ricardo; BOHN, Liana)

\section{Considerações Finais}

Investigações sobre os níveis de desenvolvimento dos países são bastante difundidas na literatura acadêmica, mas análises que visem incorporar medidas de eficiência na utilização dos recursos envolvidos nesse processo ainda são incipientes. Visando ocupar uma fração dessa lacuna, o presente trabalho procurou mensurar a eficiência de 66 nações na promoção do bem estar, e incorporá-las no cômputo de um novo indicador de desenvolvimento. Para tanto, leva-se em conta não somente as dimensões comumente envolvidas no cômputo do IDH, mas também uma medida da eficiência ambiental. Uma das contrapartidas do processo de desenvolvimento se dá em termos de externalidades ambientais negativas, de modo que é importante levar em consideração em que medida estas são compensadas pelos ganhos de bem estar. Tal análise fundamenta-se na ideia de que é preciso buscar o desenvolvimento, mas minimizando o impacto sobre o meio ambiente, condição necessária para que as nações continuem a se desenvolver no futuro.

Os resultados ora apresentados permitem mensurar e comparar as eficiências de cada país em termos de seus gastos com educação e saúde, bem como na emissão de carbono, além de fornecer uma medida alternativa de desenvolvimento que considere tais medidas. Assim, é possível observar que países de renda e tecnologia mais elevadas tendem a ser mais eficientes na utilização de seus recursos, o que lhes dá uma vantagem não apenas no presente, mas também no médio e longo prazo. Países em desenvolvimento tendem a padecer mais de problemas de má gestão e má aplicação das tecnologias disponíveis - provavelmente por ainda não possuírem acesso às tecnologias mais eficientes -, que se colocam como uma barreira ao processo de catching-up. Desencadear um processo de desenvolvimento sustentado envolve, portanto, superar esses problemas, tarefa cujos meios e métodos ensejam uma compreensão de que a aplicação de recursos deve sempre envolver esforços de quantificação, tanto dos gastos quanto dos impactos, e que esse processo, quando publicamente conduzido, deve primar pelo máximo de transparência e accountability. 
Artigo | Índice de Desenvolvimento Humano Eficiente e Sustentável (IDHES): Uma Proposta Alternativa (SOARES, Thiago Costa; DALBERTO, Cassiano Ricardo; BOHN, Liana)

\section{Referências}

BANKER, Rajiv D.; CHARNES, Abraham; COOPER, William Wager. Some models for estimating technical and scale inefficiencies in data envelopment analysis. Management science, v. 30, n. 9, p. 1078-1092, 1984.

CHIU, Ching-Ren et al. Decomposition of the environmental inefficiency of the metafrontier with undesirable output. Energy Economics, v. 34, n. 5, p. 1392-1399, 2012.

CHUNG, Yangho H.; FÄRE, Rolf; GROSSKOPF, Shawna. Productivity and undesirable outputs: a directional distance function approach. journal of Environmental Management, $v$. 51, n. 3, p. 229-240, 1997.

COSTANTINI, Valeria; MONNI, Salvatore. Sustainable human development for European countries. Journal of Human Development, v. 6, n. 3, p. 329-351, 2005.

DALBERTO, Cassiano Ricardo et al. Índice de Desenvolvimento Humano Eficiente: uma mensuração alternativa do bem-estar das nações. 2015.

FÄRE, Rolf et al. Characteristics of a polluting technology: theory and practice. journal of Econometrics, v. 126, n. 2, p. 469-492, 2005.

HICKS, Norman; STREETEN, Paul. Indicators of development: the search for a basic needs yardstick. World development, v. 7, n. 6, p. 567-580, 1979.

HICKS, Douglas A. The inequality-adjusted human development index: a constructive proposal. World development, v. 25, n. 8, p. 1283-1298, 1997.

IYER, K.; RAMBALDI, A.; TANG, K. K. Globalisation and the Technology Gap: Regional and Time Evidence, Leading Economic and Managerial Issues Involving Globalisation. Nova Science New York, 2006. 213-227.

KEYES, Corey LM. Subjective well-being in mental health and human development research worldwide: An introduction. Social indicators research, v. 77, n. 1, p. 1-10, 2006.

MACCARI, Norma. Sustainable human development: Human Development Index and the environment. International Journal of Sustainable Human Development, v. 2, n. 1, p. 2934, 2014.

NORTH, D. C. Institutions, institutional change and economic performance. Cambridge: Cambridge University Press, 1990.

O'DONNELL, Christopher J.; RAO, DS Prasada; BATTESE, George E. Metafrontier frameworks for the study of firm-level efficiencies and technology ratios. Empirical economics, v. 34, n. 2, p. 231-255, 2008.

$\mathrm{OH}$, Dong-hyun; LEE, Jeong-dong. A metafrontier approach for measuring Malmquist productivity index. Empirical economics, v. 38, n. 1, p. 47-64, 2010.

OLIVEIRA, P. S. ebold et al. Os índices de bem estar e felicidade como alternativas para a mensuração do desenvolvimento dos países. DEBATESVII, p. 61, 2016.

STANTON, Elizabeth A. The human development index: A history. PERI Working Papers, p. 85, 2007.

TÜRE, Cengiz. A methodology to analyse the relations of ecological footprint corresponding with human development index: eco-sustainable human development index. International Journal of Sustainable Development \& World Ecology, v. 20, n. 1, p. 9-19, 2013.

UNITED NATIONS DEVELOPMENT PROGRAMME - UNDP - Human Development Report 2015. New York: PBM Graphics, 2015. 
Artigo | Índice de Desenvolvimento Humano Eficiente e Sustentável (IDHES): Uma Proposta Alternativa (SOARES, Thiago Costa; DALBERTO, Cassiano Ricardo; BOHN, Liana)

UNRUH, Gregory C. Understanding carbon lock-in. Energy policy, v. 28, n. 12, p. 817$830,2000$.

URA, Karma et al. The GNH Index. In: 4th International Gross National Happiness Conference, Thimphu. 2008.

WORLD BANK. World Bank Group, 2015. Disponivel em: <http://www.worldbank.org/>. Acesso em: Janeiro 2015.

ZHANG, Ning; CHOI, Yongrok. Total-factor carbon emission performance of fossil fuel power plants in China: A metafrontier non-radial Malmquist index analysis. Energy Economics, v. 40, p. 549-559, 2013. 